\title{
Replication protein A is an independent prognostic indicator with potential therapeutic implications in colon cancer
}

\author{
Nikolaos Givalos ${ }^{1}$, Hariklia Gakiopoulou ${ }^{2}$, Melina Skliri ${ }^{2}$, Katerina Bousboukea ${ }^{2}$, \\ Anastasia E Konstantinidou ${ }^{2}$, Penelope Korkolopoulou ${ }^{2}$, Maria Lelouda ${ }^{2}$, \\ Gregory Kouraklis ${ }^{1}$, Efstratios Patsouris ${ }^{2}$ and Gabriel Karatzas ${ }^{1}$ \\ ${ }^{1}$ Department of Surgery, Medical School, National Kapodistrian University of Athens, Athens, Greece and \\ ${ }^{2}$ Department of Pathology, Medical School, National Kapodistrian University of Athens, Athens, Greece
}

\begin{abstract}
Replication protein A (RPA), a component of the origin recognition complex, is required for stabilization of single-stranded DNA at early and later stages of DNA replication being thus critical for eukaryotic DNA replication. Experimental studies in colon cancer cell lines have shown that RPA protein may be the target of cytotoxins designed to inhibit cellular proliferation. This is the first study to investigate the expression of RPA1 and RPA2 subunits of RPA protein and assess their prognostic value in colon cancer patients. We analyzed immunohistochemically the expression of RPA1 and RPA2 proteins in a series of 130 colon cancer resection specimens in relation to conventional clinicopathological parameters and patients' survival. Statistical significant positive associations emerged between: (a) RPA1 and RPA2 protein expressions $(P=0.0001)$, (b) RPA1 and RPA2 labelling indices (LIs) and advanced stage of the disease $(P=0.001$ and 0.003 , respectively), (c) RPA1 and RPA2 LIs and the presence of lymph node metastasis ( $P=0.002$ and 0.004 , respectively), (d) RPA1 LI and the number of infiltrated lymph nodes $(P=0.021),(e)$ RPA2 LI and histological grade of carcinomas $(P=0.05)$. Moreover, a statistical significant higher RPA1 LI was observed in the metastatic sites compared to the original ones $(P=0.012)$. RPA1 and RPA2 protein expression associated with adverse patients' outcome in both univariate (log rank test: $\boldsymbol{P}<\mathbf{0 . 0 0 0 0 1}$ and $\mathbf{0 . 0 0 0 0 1}$, respectively) and multivariate (Cox model: $P=0.092$ and $\mathbf{0 . 0 0 0 1}$, respectively) statistical analysis. Statistical significant differences according to the expression of RPA1 and RPA2 proteins were also noticed in the survival of stage II $(P<0.00001$ and 0.0016 , respectively) and stage III $(P=0.0029$ and 0.0079 , respectively) patients. In conclusion, RPA1 and RPA2 proteins appear to be useful prognostic indicators in colon cancer patients and attractive therapeutic targets for regulation by tumor suppressors or other proteins involved in the control of cell proliferation. Modern Pathology (2007) 20, 159-166. doi:10.1038/modpathol.3800719
\end{abstract}

Keywords: replication protein $A$; colon cancer; survival

The proper functioning of the replication machinery is a prerequisite for the maintenance of genomic integrity. Thus, doubling of the mammalian genome is performed with an accuracy of only one misincorporation per $1010 \mathrm{bp} .^{1}$ In tumor cells, the accuracy of replication appears to be disturbed as not only numerous single-nucleotide mutations but also chromosomal changes are accumulated in the genome during tumor progression. ${ }^{2}$

Correspondence: Dr N Givalos, MD, PhD, Department of Surgery, Medical School, National Kapodistrian University of Athens, 5 Iras str., P Psyhiko, GR-154 52 Athens, Greece.

E-mail: givalos@teledomenet.gr

Received 17 April 2006; revised 16 June 2006; accepted 26 September 2006
Replication protein A (RPA), a component of the origin recognition complex, is required for stabilization of single-stranded DNA at early and later stages of DNA replication being thus critical for eukaryotic DNA replication. ${ }^{3,4}$ Moreover, RPA is involved in DNA recombination and repair. ${ }^{5-8}$ Additionally, it has also been implicated in the regulation of apoptosis and gene expression. ${ }^{9}$ Links between RPA and transcription have been postulated based on the observed interactions between RPA and Gal4, VP16, p53, Stat3, RBT1 and menin. ${ }^{10-15}$ This protein is present in cells as a heterotrimeric complex consisting of three subunits which, in order of decreasing size, have been designated as RPA1 (70 kDa), RPA2 (32 kDa) and RPA3 $(14 \mathrm{kDa}) .{ }^{16-19}$ Recently, RPA complex has been identified as an autoantigen in breast cancer patients thus adding 
another member to the growing list of cancer autoantigens known to play important role in cell proliferation..$^{20,21}$ Moreover, experimental studies in colon cancer cell lines have shown that RPA protein may be the target of cytotoxins designed to inhibit cellular proliferation. ${ }^{22}$ However, there is no previous study investigating RPA immunohistochemical expression in human colon cancer specimens.

The aim of the present study was to investigate the immunohistochemical expression of RPA1 and RPA2 subunits of the RPA protein in a series of 130 colon cancer resection specimens. Moreover, RPA1 and RPA2 expression was analyzed in relation to conventional clinicopathological parameters and patients' survival.

\section{Materials and methods}

\section{Patients}

This is a retrospective study of 130 consecutive patients who presented with primary colon carcinomas between 1988 and 2003, for whom paraffinembedded tissue and clinical information were available. There were 60 men and 70 women with a median age of 58 years (range 29-87 years). None of the patients had received chemotherapy or radiation before surgery. Tumor size ranged from 2.2 to $14 \mathrm{~cm}$ (median value: $5.5 \mathrm{~cm}$ ). According to the sixth edition of the TNM system of cancer staging adopted by the American Joint Committee on Cancer and the International Union Against Cancer (AJCC/IUCC), tumors were classified as stage I: seven cases (four T1N0M0 and three T2N0M0 cases), stage II: 70 cases (62 stage IIA (T3N0M0) and eight stage IIB (T4N0M0) cases), stage III: 41 cases (three stage IIIA (T1-T2N1M0), 14 stage IIIB (T3T4N1M0) and 24 stage IIIC (anyTN2M0) cases) and 12 stage IV (Any T Any N M1) cases. All cases were reviewed and assigned a histologic grade according to WHO classification of colon carcinomas: there were 13 grade 1,98 grade 2 and 19 grade 3 carcinomas. Follow-up period ranged from 2 to 180 months (mean: 43 months, median: 30 months). During this period, 38 disease-specific deaths were recorded. The clinicopathologic characteristics of our patients are shown in Table 1.

\section{Processing of Specimens and Immunohistochemistry}

Tissues were fixed immediately after removal in $10 \%$ buffered formalin and processed to paraffin wax. Four $\mu \mathrm{m}$ serial sections were cut from each specimen on superfrost plus glass slides and left to dry overnight at $37^{\circ} \mathrm{C}$. Immunohistochemical detection of RPA1 and RPA2 proteins was performed by standard streptavidin peroxidase method using the monoclonal antibodies anti-RPA1 (P70 subunit, NA13, oncogene) and anti-RPA2 (P32 subunit, NA18, oncogene) in a dilution of 1:50 for each
Table 1 Clinicopathologic characteristics of 130 colon carcinoma patients

\begin{tabular}{|c|c|c|}
\hline \multicolumn{3}{|l|}{ Age at surgery (years) } \\
\hline Median (mean) & $58(58.4)$ & \\
\hline Range & $29-84$ & \\
\hline \multicolumn{3}{|l|}{ Sex } \\
\hline Male & 60 & \\
\hline Female & 70 & \\
\hline \multicolumn{3}{|l|}{ Tumor size $(\mathrm{cm})$} \\
\hline Median (mean) & $5.5(5.9)$ & \\
\hline Range & $2.2-14$ & \\
\hline \multicolumn{3}{|l|}{ Tumor stage } \\
\hline I & 7 & $5.4 \%$ \\
\hline II & 70 & $53.8 \%$ \\
\hline III & 41 & $31.5 \%$ \\
\hline IV & 12 & $9.2 \%$ \\
\hline \multicolumn{3}{|l|}{ Tumor grade } \\
\hline 1 & 13 & $9.8 \%$ \\
\hline 2 & 98 & $75.6 \%$ \\
\hline 3 & 19 & $14.6 \%$ \\
\hline \multicolumn{3}{|l|}{ Follow-up statistics } \\
\hline Mean (months) & 43.3 & \\
\hline Median (months) & 30 & \\
\hline Range & $2-180$ & \\
\hline Deaths due to colon cancer & 38 & \\
\hline
\end{tabular}

antibody. To enhance antigen retrieval sections underwent microwave treatment (using 1 Mm EDTA $\mathrm{PH}$ 8.0). Staining for all antibodies was assessed blindly (ie without any knowledge of the clinical data) by two observers. Whenever a difference of greater than $5 \%$ between the two assessments was observed, slides were reviewed jointly and a consensus was reached. Nuclei from about 1000 tumor cells from systematically randomized fields $(\times 40)$ throughout the entire section were counted and the labelling index (LI) was calculated as the percentage of labelled nuclei out of the total number of tumor cells counted. Whenever heterogeneous staining was encountered, counting was performed in areas of highest density of labelled cells, identified at medium magnification $(\times 20)$, because it has been proposed that these tumor areas are most likely to be of biological significance. All clearly identifiable nuclear staining beyond background was recorded as positive for RPA1 and RPA2.

No lymphoid cells were included in the counts even though they expressed both RPA1 and RPA2. In 30 cases immunohistochemistry was also performed in lymph nodes with metastatic deposits in order to compare RPA1 and RPA2 expression in the original and metastatic site.

\section{Statistical Analysis}

The normality of distributions was tested with the Kolmogorov-Smirnov test. The Mann-Whitney 
$U$-test was used to compare central tendencies in the distribution of the numerical variables between tumor size categories and the presence or absence of lymph node metastasis. Associations between numerical parameters and stage, grade and type of performed surgery were assessed with KruskalWallis analysis of variance. Spearman's rank correlation coefficient was calculated to determine associations between numerical variables.

The prognostic effect of various parameters (ie age, gender, grade, stage, type of treatment, RPA1 and RPA2 expression) on clinical outcome (ie death of disease) was tested by plotting survival curves according to Kaplan-Meier method and comparing groups using the log rank test, as well as by multivariate analysis using the Cox regression model. Patients dying of other causes during the follow-up period were treated as censored data. In univariate analysis, the continuous variables (age, tumor size, RPA1 and RPA2 expression) were categorized on the basis of the median value. To avoid any 'data-driven' categorization, continuous variables were entered in multivariate analysis as continuous variables.

Statistical analysis was performed using the SPSS for Windows Software (SPSS Inc., Chicago, IL, USA). A $P$-value of less than or equal to 0.05 was considered indicative of a statistically significant difference.

\section{Results}

Immunohistochemical analysis in control normal human colon revealed that RPA1 and RPA2 immunoreactivity was present in colonic epithelial cells in the lower two-thirds of the crypts. An average of one to three positive cells per crypt was observed with nuclear localization. Positive cells were evenly dispersed and did not occur in clusters. In adenomas, most RPA1 and RPA2 immunoreactive cells were observed in the superficial mucosa, at the luminal surface of the polyps. A gradual increase in RPA1 and RPA2 immunohistochemical expression was observed as tissues evolved from normal to dysplastic and to invasive carcinoma.

In carcinomas, immunoreactivity for RPA1 and RPA2 proteins was seen in all cases ranging from 2 to $83 \%$, with a median of 35 and $12 \%$, respectively. The pattern of staining was mostly nuclear, although a faint cytoplasmic staining was seen in a few cases, which was disregarded as non-specific (Figures 1 and 2). Nuclear staining was strong to moderate in almost all cases investigated. Thus, grading of staining intensity using a semiquantitative method was not applicable. A strong positive correlation emerged between RPA1 and RPA2 expressions (Spearman's $\rho=0.809, P=0.0001$ ).

When analyzing the relationship between RPA1 or RPA2 expression and stage, a significant difference

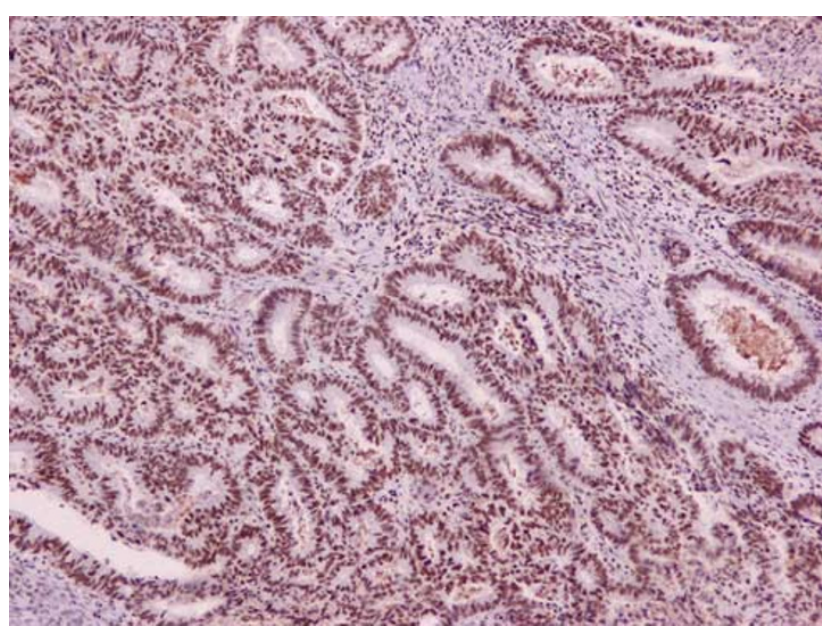

Figure 1 RPA1 immunohistochemical expression in numerous neoplastic nuclei of a colon carcinoma.

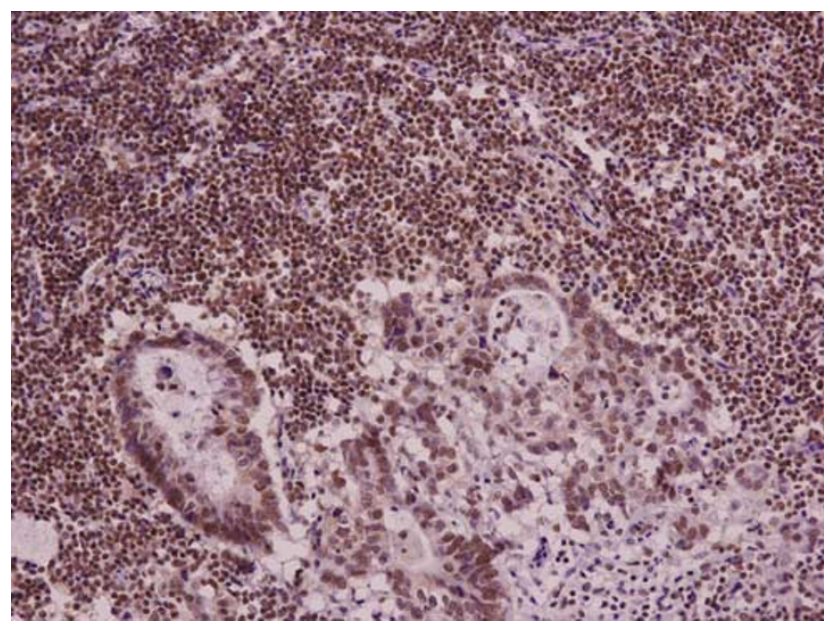

Figure 2 RPA1 immunoreactivity in metastatic colon cancer deposits from an infiltrated lymph node.

was registered, inasmuch as high stage tumors displayed a higher expression level than the remaining cases (Kruskal-Wallis test: $P=0.001$ and 0.003 for RPA 1 and RPA2 and tumor stage, respectively) (Figure 3). Moreover, RPA1 and RPA2 labelling indices (LIs) were significantly correlated with the presence of lymph node metastasis (MannWhitney $U$-test: $P=0.002$ and 0.004 , respectively, Figure 4) and RPA1 LI with the number of infiltrated lymph nodes (Spearman's $\rho=0.419, P=0.021$ ). On the other hand, neither RPA1 nor RPA2 associated significantly with tumor size. An interesting finding of this study lies on the observation of increased RPA1 expression in the metastatic sites compared to the original ones (Wilcoxon signed ranks test: $P=0.012$ ). Finally, a significant positive association was established between RPA2 LI and increased grade of the disease (Kruskal-Wallis test: $P=0.05$, Figure 5). 


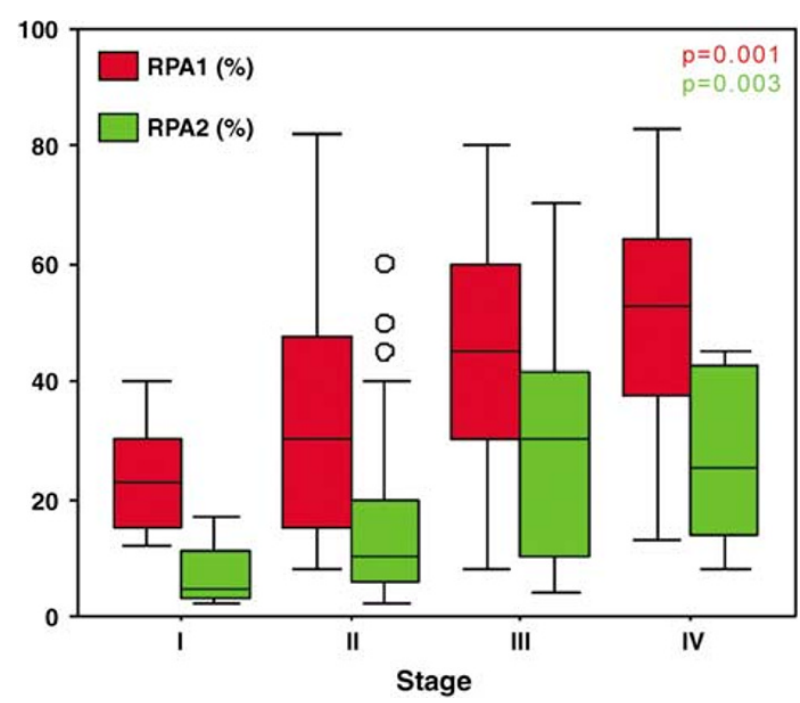

Figure 3 RPA1 and RPA2 LIs in relation to stage of the disease $(P=0.001$ and 0.003 , respectively)

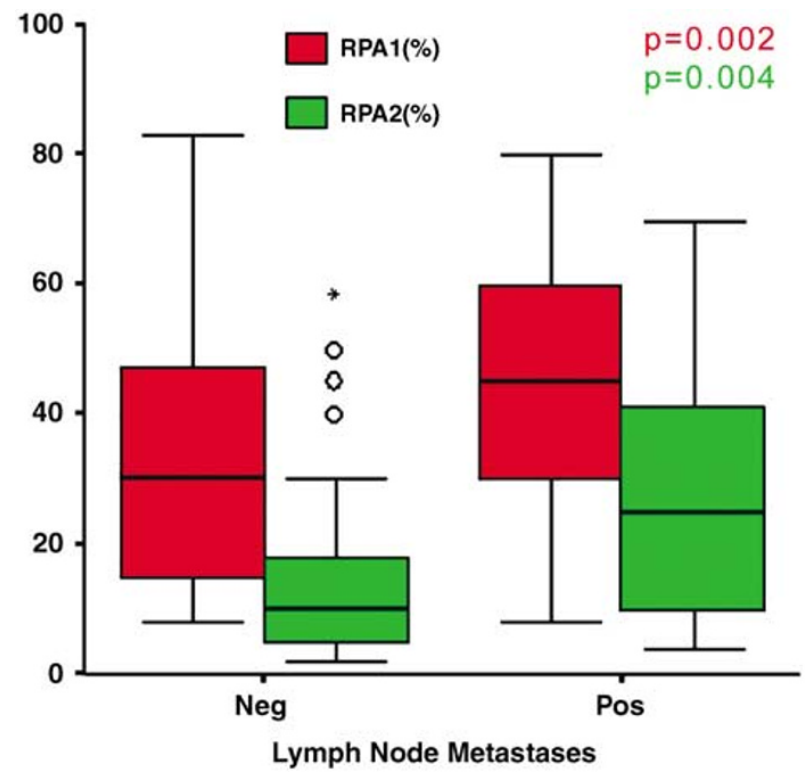

Figure 4 RPA1 and RPA2 LIs in relation to lymph node metastases $(P=0.002$ and 0.004 , respectively).

Univariate survival analysis demonstrated that patients with high RPA1 and RPA2 LIs, categorized on the basis of their median values (ie 35 and $12 \%$, respectively), had a significantly worse overall survival compared to patients with low RPA1 and RPA2 LIs (log rank test: $P<0.00001$ for each protein, Figures 6 and 7). Mean survival time of patients with RPA1 LI less than $35 \%$, was 173 months compared to 39 months of patients with RPA1 LI greater than $35 \%$ (Figure 6). Two deaths were recorded among the 54 patients of the former group compared to 36 deaths recorded among the 50 patients of the later group (Figure 6). None of the patients with RPA2 LI less than $12 \%$ died from disease whereas 21 out of

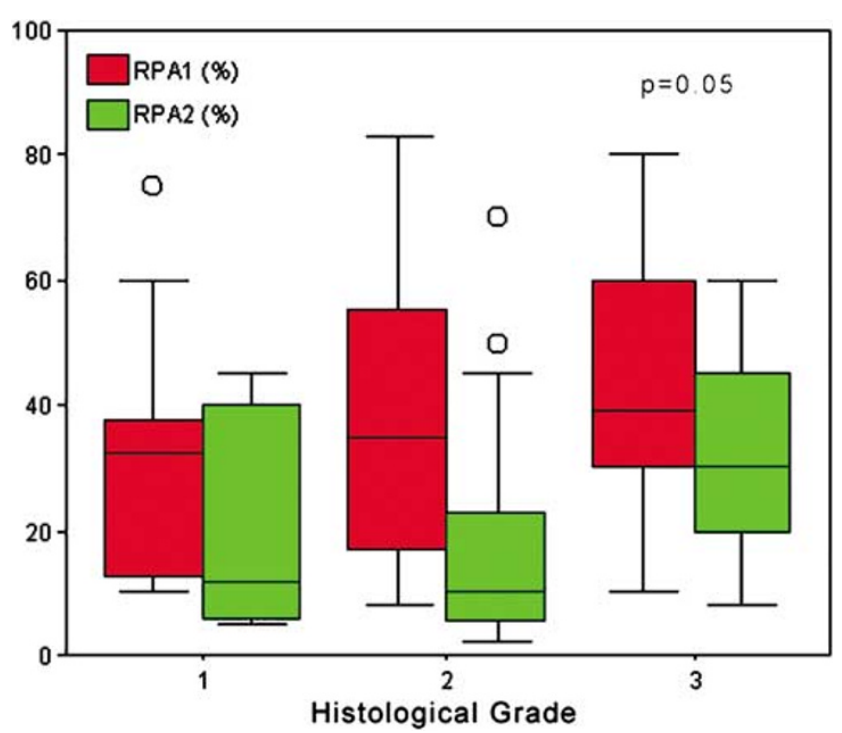

Figure 5 RPA1 and RPA2 LIs in relation to tumor grade $(P=0.05$ for RPA2).

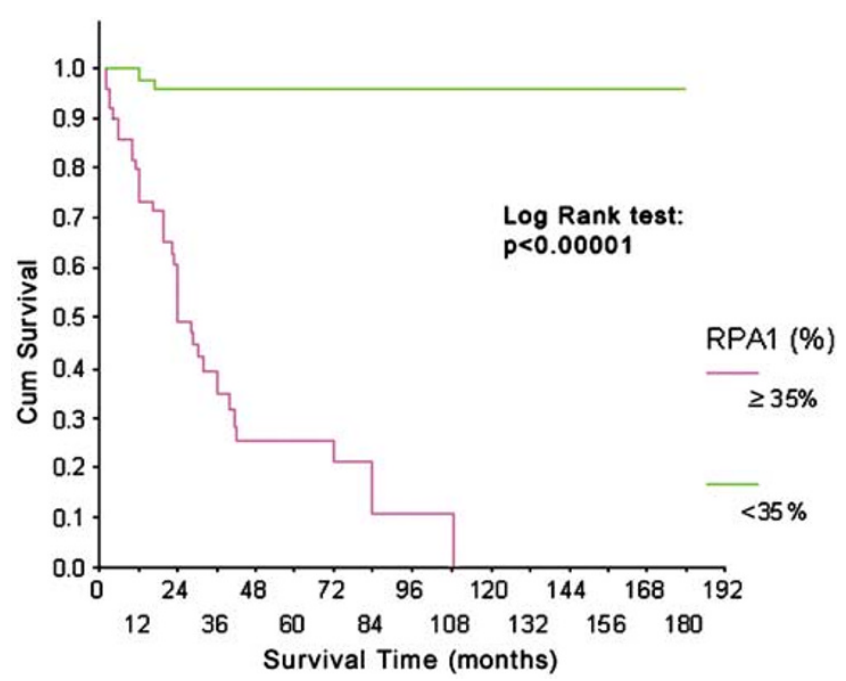

Figure 6 Kaplan-Meier overall survival curves according to the immunohistochemical expression of RPA1 protein categorized on the basis of the median value (35\%). Increased (>35\%) RPA1 expression is significantly associated with shorter overall survival $(P<0.00001)$.

37 patients with RPA2 LI greater than $12 \%$ were dead during the follow-up period (Figure 7). In order to test the value of RPA1 and RPA2 as prognosticators in patients of the same stage category, we stratified patients according to stage and we performed survival analysis according to the expression of RPA1 and RPA2. Statistical significant associations emerged between high RPA1 and RPA2 LIs and poor overall survival of patients with stage II $(P<0.00001$ and 0.0016 , respectively) as well as of patients with stage III $(P=0.0029$ and 0.0079 , respectively) disease (Figures 8 and 9).

In multivariate analysis (Cox regression model), RPA2 protein expression emerged as an independent 
predictor of survival $(P<0.0001)$ along with stage of the disease $(P=0.002)$ (Table 2). Moreover, an association of suggestive significance was established between RPA1 LI and patient survival $(P=0.092)$ (Table 2).

\section{Discussion}

Evaluation of cell cycle regulators has gained special interest in the effort to increase the amount of prognostic information in malignant tumors. RPA is a highly conserved, single-stranded DNA-binding multisubunit protein complex involved in eukaryotic DNA replication, recombination and repair. ${ }^{3,5}$ Previous experimental studies in colon cancer cell lines have reported a reduction in DNA replication

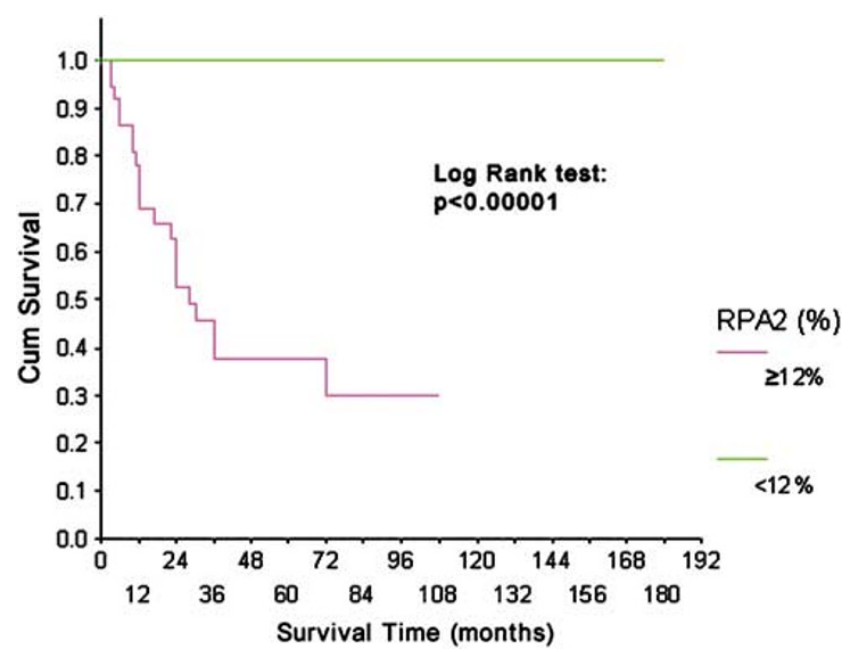

Figure 7 Kaplan-Meier overall survival curves according to the immunohistochemical expression of RPA2 protein categorized on the basis of the median value $(12 \%)$. Increased $(>12 \%)$ RPA2 expression is significantly associated with shorter overall survival $(P<0.00001)$. activity in parallel with the reduction of RPA1 protein suggesting a crucial role of RPA complex for the proliferative activity of colon cancer cells. ${ }^{14}$ However, there is no previous study investigating the in vivo expression of RPA proteins in human colon cancer tissues.

In the present study, we have clearly demonstrated by immunohistochemistry the nuclear expression of RPA1 and RPA2 proteins in a series of colon carcinomas. This is in keeping with cell fractionation studies indicating that RPA is almost exclusively nuclear. ${ }^{23}$ Moreover, a widespread expression was observed in agreement with the reported abundant RPA staining in breast cancer cells. ${ }^{20}$ RPA protein overexpression in tumor cells does not seem to result from mutation of the encoding gene since Popanda et $a{ }^{24}$ failed to detect amino-acid changes in the RPA and other DNA replication genes analyzed in patients with sporadic colon cancer. ${ }^{25}$ The authors have suggested that this scarcity of mutations is due to stringent selection, eliminating functionally impaired replication proteins. ${ }^{24}$ The mechanism of RPA1 and RPA2 protein overexpression in colon carcinomas merits further investigation. The significant association observed between RPA1 and RPA2 protein expression reflects the similar kinetics of these proteins, being upregulated in order to accomplish their potential proliferative task during cancer progression.

According to our results, both RPA1 and RPA2 LIs were significantly associated with advanced stage of the disease and lymph node metastasis suggesting a role of these proteins in colon cancer progression, probably explained on the basis of tumor growth selection for the most rapidly proliferating cells. This concept is further strengthened by the observation in our study significantly higher expression of RPA1 protein in the metastatic sites compared to the original ones. On the other hand, the apparent increase of DNA stabilizing proteins such as RPA1 and RPA2 in cancer, a situation characterized by
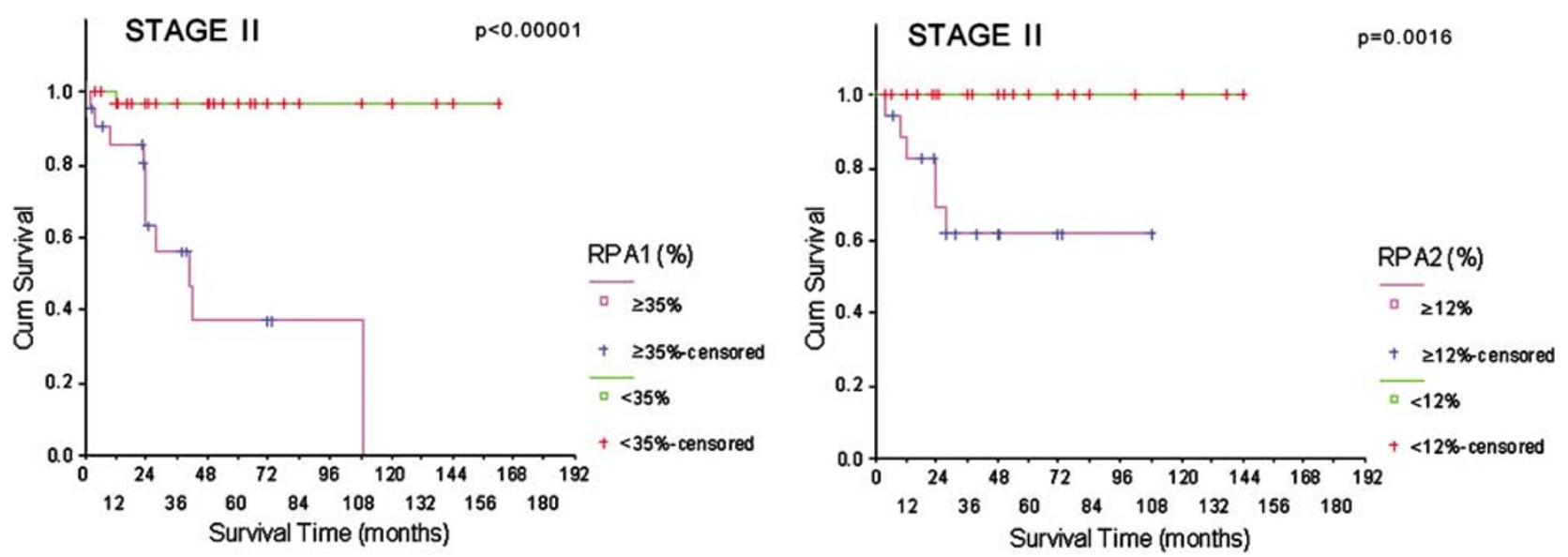

Figure 8 Kaplan-Meier overall survival curves in stage II patients, according to the immunohistochemical expression of RPA1 and RPA2 proteins categorized on the basis of their median values. Increased RPA1 or RPA2 protein expression is significantly associated with poor survival of stage II patients $(P<0.00001$ and 0.0016 , respectively). 

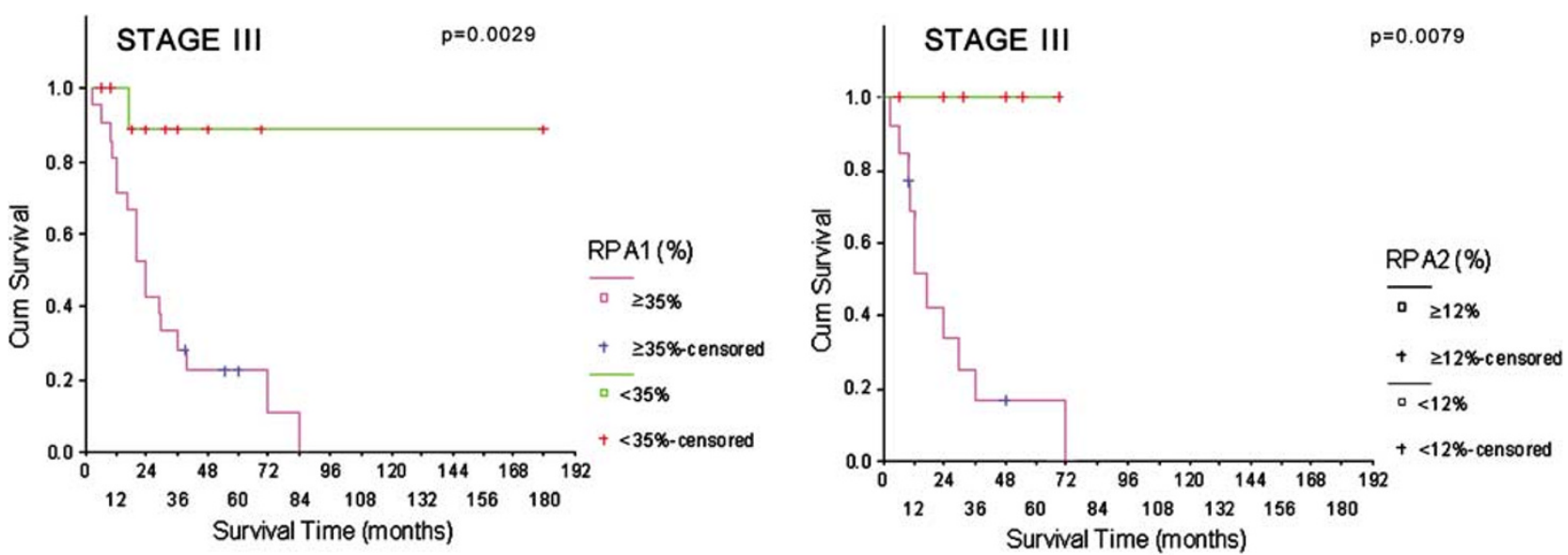

Figure 9 Kaplan-Meier overall survival curves in stage III patients, according to the immunohistochemical expression of RPA1 and RPA2 proteins categorized on the basis of their median values. Increased RPA1 or RPA2 protein expression is significantly associated with poor survival of stage III patients $(P=0.0029$ and 0.0079 , respectively).

Table 2 Multivariate survival analysis (Cox proportional hazard model)

\begin{tabular}{|c|c|c|c|c|c|c|c|c|}
\hline & \multirow[t]{2}{*}{$B$} & \multirow[t]{2}{*}{ s.e. } & \multirow[t]{2}{*}{ Wald } & \multirow[t]{2}{*}{$d f$} & \multirow[t]{2}{*}{ Sig. } & \multirow[t]{2}{*}{$\operatorname{Exp}(B)$} & \multicolumn{2}{|c|}{$95.0 \%$ CI for $\operatorname{Exp}(B)$} \\
\hline & & & & & & & Lower & Upper \\
\hline \multicolumn{9}{|c|}{ Variables in the equation } \\
\hline Stage & 1.214 & 0.395 & 9.456 & 1 & 0.002 & 3.368 & 1.553 & 7.304 \\
\hline RPA2 & 0.067 & 0.013 & 28.057 & 1 & 0.000 & 1.069 & 1.043 & 1.096 \\
\hline & Score & $d f$ & Sig. & & & & & \\
\hline \multicolumn{9}{|c|}{ Variables not in the equation ${ }^{\mathrm{a}}$} \\
\hline Age & 2.018 & 1 & 0.155 & & & & & \\
\hline Grade & 1.119 & 1 & 0.290 & & & & & \\
\hline RPA1 & 2.836 & 1 & 0.092 & & & & & \\
\hline
\end{tabular}

${ }^{\mathrm{a}}$ Residual $\chi^{2}=5.549$ with $3 \mathrm{df}$, Sig. $=0.136$.

increased destabilization of the genome, would appear to be contradictory. Indeed, apart from its crucial role in the proper functioning of the replication machinery, RPA protein is involved in DNA damage recognition and repair. RPA is a key factor of a highly versatile and sophisticated DNA damage removal pathway called 'nucleotide excision repair' (NER) that counteracts the deleterious effects of a multitude of DNA lesions. ${ }^{8}$ Thus, one could postulate that the significant increase of RPA in carcinomas and particularly in high grade and advanced stage carcinomas might indicate a tendency to develop a feedback mechanism to come up to the increasing need for DNA repair synthesis. However, the exact functions of RPA1 and RPA2 during the neoplastic process merits further investigation.

The most interesting finding of this study lies on the survival analysis results. RPA1 and RPA2 proteins emerged as significant prognosticators of adverse outcome in univariate analysis. In multivariate analysis, RPA2 protein emerged as a significant independent predictor of survival along with stage of the disease whereas RPA1 also affected survival independently with a suggestion, however, valuable. The confirmation of the adverse prognostic effect of stage, a universally established prognostic factor, proves that our cohort was representative and that survival analysis was valid. The independent prognostic value of RPA2 protein might suggest an underlying mechanism by which changes in RPA2 could facilitate tumor progression. RPA2 is capable of complex formation with the tumor-suppressor protein p53 modulating the p53-mediated DNA damage checkpoint response by sequestering p53 and preventing the transcriptional activation of genes involved in DNA repair. ${ }^{26,27}$ It has been proposed that RPA2 overexpression might effectively inactivate p53, thus allowing cells with damaged DNA to proceed through the cell cycle. ${ }^{20}$ Specific interactions have also been demonstrated between RPA2 and menin, another tumor-suppressor protein, whose gene-MEN1-is disrupted in multiple endocrine neoplasia type 1 and which is postulated to participate in the maintenance of genomic integrity. ${ }^{15}$ However, further investigation 
is required to determine the significance of this interaction.

To further test the prognostic value of RPA proteins and their possible implication in the therapeutic decision-making, we performed survival analyzes stage by stage. In stage II as well as in stage III patients, survival was statistically, significantly different depending on the expression levels of either RPA1 or RPA2 protein. These findings are of particular interest especially for stage II patients in which most therapeutic dilemmas are raised.

The role of RPA1 and RPA2 proteins may not be restricted to that of prognostic index. Experimental studies in colon cancer cell lines have shown that RPA protein may be the target of cytotoxins designed to inhibit cellular proliferation. ${ }^{22}$ It has been reported that tirapazamine (TPZ), a hypoxia-selective cytotoxin which is currently being examined in phase II and III clinical trials, greatly inhibits DNA synthesis and thus cell proliferation, through direct damage to or intracellular redistribution of RPA protein. ${ }^{28-32}$ Using an in vitro DNA replication assay, Peters et $a^{22}$ observed that extracts from TPZ-treated colon cancer cells had a reduced ability to support in vitro DNA replication which was accompanied by a reduction in the levels of RPA. Addition of recombinant RPA restored replication activity to control levels. Consequently, the observation in this study indicates widespread expression of RPA1 and RPA2 proteins along with their prognostic implications for colon cancer patients, suggest that apart from their putative application as prognostic indicators, they could be an attractive target for regulation by tumor-suppressors or other proteins involved in the control of cell proliferation. ${ }^{15}$ Indeed and as we mentioned previously, specific interaction between p53 and RPA is inversely correlated with the abilities of RPA to bind ssDNA and of p53 to bind transactivating sequences in the promoters of its target genes. ${ }^{12,26}$ This interaction is disrupted by UV, adozelesin and cisplatin and a correlation between RPA-p53-binding and RPA2 phosphorylation status has been observed. ${ }^{33,34}$ RPA2 has been shown to undergo cell cycle-dependent and damage-induced phosphorylation, and although the consequences of this phosphorylation are unclear, it may influence RPA conformation, DNA-binding characteristics, and/or its interactions with other proteins. $^{33,34}$

In conclusion, the widespread expression of RPA1 and RPA2 proteins in colon carcinomas suggests that these proteins are implicated in colon cancer growth. Moreover, their association with advanced stage, lymph node metastasis as well as their increased expression in metastatic sites supports a role in cancer progression. However, the exact role of these multifunctional molecules in the neoplastic process merits further investigation. According to our results, RPA1 and RPA2 emerged as predictors of poor overall survival, implying that these proteins may prove to be useful prognostic markers in patients with colon carcinomas and especially in patients with stage II and III carcinomas. Finally, the role of RPA1 and RPA2 proteins may not be limited to that of a prognostic marker but may direct avenues of research and therapeutic interventions.

\section{References}

1 Loeb LA. Cancer cells exhibit a mutator phenotype. Adv Cancer Res 1998;72:25-56.

2 Nowell PC. Mechanisms of tumor progression. Cancer Res 1986;46:2203-2207.

3 Hübscher U, Maga G, Podust VN. DNA replication accessory proteins. In: De Pamphilis ML (ed). DNA Replication in Eukaryotic Cells. Cold Spring Harbor Laboratory Press, Cold Spring Harbor: New York, 1996, pp 525-543.

4 Bochkarev A, Pfuetzner RA, Edwards AM, et al. Structure of the singlestranded DNA-binding domain of replication protein A bound to DNA. Nature (London) 1997;385:176-181.

5 He Z, Henricksen LA, Wold MS, et al. RPA involvement in the damage recognition and incision steps of nucleotide excision repair. Nature (London) 1995; 374:566-569.

$6 \mathrm{Li} \mathrm{L}, \mathrm{Lu}$ X, Peterson CA, et al. An interaction between the DNA repair factor XPA and replication protein A appears essential for nucleotide excision repair. Mol Cell Biol 1995;15:5396-5402.

7 Singh KK, Samson L. Replication protein A binds to regulatory elements in yeast DNA repair and DNA metabolism genes. Proc Natl Acad Sci USA 1995;92: 4907-4911.

8 de Laat WL, Jaspers NGJ, Hoeijmakers JHJ. Molecular mechanism of nucleotide excision repair. Genes Dev 1999;13:768-785.

9 Iftode C, Daniely Y, Borowiec JA. Replication protein A (RPA): the eukaryotic SSB. Crit Rev Biochem Mol Biol 1999;34:141-180.

10 He Z, Brinton BT, Greenblatt J, et al. The transactivator proteins VP16 and GAL4 bind replication factor A. Cell 1993;73:1223-1232.

11 Li R, Botchan MR. The acidic transcriptional activation domains of VP16 and p53 bind the cellular replication protein $\mathrm{A}$ and stimulate in vitro $\mathrm{BPV}-1$ DNA replication. Cell 1993;73:1207-1221.

12 Dutta A, Ruppert JM, Aster JC, et al. Inhibition of DNA replication factor RPA by p53. Nature 1993;365:79-82.

13 Kim J, Kim D, Chung J. Replication protein A 32 kDa subunit (RPA p32) binds the SH2 domain of Stat3 and regulates its transcriptional activity. Cell Biol Int 2000;24:467-473.

14 Cho JM, Song DJ, Bergeron J, et al. RBT1, a novel transcriptional co-activator, binds the second subunit of replication protein A. Nucleic Acids Res 2000;28: 3478-3485.

15 Sukhodolets KE, Hickman AB, Agarwal SK, et al. The 32-kilodalton subunit of replication protein a interacts with menin, the product of the MEN1 tumor suppressor gene. Mol Cell Biol 2003;23:493-509.

16 Wold MS. Replication protein A: a heterotrimeric, single-stranded DNA-binding protein required for eukaryotic DNA metabolism. Ann Rev Biochem 1997; 66:61-92. 
17 Erdile LF, Heyer WD, Kolodner R, et al. Characterization of a cDNA encoding the 70-kDa single-stranded DNA-binding subunit of human replication protein $\mathrm{A}$ and the role of the protein in DNA replication. J Biol Chem 1991;266:12090-12098.

18 Erdile LF, Wold MS, Kelly TJ. The primary structure of the 32-kDa subunit of human replication protein A. J Biol Chem 1990;265:3177-3182.

19 Moore SP, Erdile L, Kelly T, et al. The human homologous pairing protein HPP-1 is specifically stimulated by the cognate single-stranded binding protein hRP-A. Proc Natl Acad Sci USA 1991;88: 9067-9071.

20 Tomkiel JE, Alansari H, Tang N, et al. Autoimmunity to the $M r 32,000$ subunit of replication protein a in breast cancer. Clin Cancer Res 2002;8:752-758.

21 Fernández-Madrid F, Tomkiel JE. Antinuclear antibodies as potential markers in the diagnosis and prognosis of lung cancer. In: Shoenfeld Y, Gershwin ME (eds). Cancer and Autoimmunity. Elsevier Science: CA, USA, 2000, pp 151-158.

22 Peters KB, Wang H, Brown JM, et al. Inhibition of DNA replication by tirazapamine. Cancer Res 2001;61: 5425-5431.

23 Treuner K, Eckerich C, Knippers R. Chromatin association of replication protein A. J Biol Chem 1998;273: 31744-31750.

24 Popanda O, Zheng C, Magdeburg JR, et al. Mutation analysis of replicative genes encoding the large subunits of DNA polymerase a and replication factors $\mathrm{A}$ and $\mathrm{C}$ in human sporadic colorectal cancers. Int J Cancer 2000;86:318-324.

25 Flohr T, Dai J-C, Büttner J, et al. Detection of mutations in the DNA polymerase $\delta$ gene of human sporadic colorectal cancers and colon cancer cell lines. Int J Cancer 1999;80:919-929.

26 Miller SD, Moses K, Jayaraman L, et al. Complex formation between p53 and replication protein A inhibits the sequence-specific DNA binding of p53 and is regulated by single-stranded DNA. Mol Cell Biol 1997;17:2101-2194.

$27 \mathrm{Li} \mathrm{R}$, Botchan MR. The acidic transcriptional activation domains of VP16 and p53 bind the cellular replication protein $\mathrm{A}$ and stimulate in vitro BPV-1 DNA replication. Cell 1993;73:1207-1221.

28 von Pawel J, von Roemeling R, Gatzemeier U, et al. Tirapazamine plus cisplatin versus cisplatin in advanced non-small-cell lung cancer: a report of the international CATAPULT I study group. Cisplatin and tirapazamine in subjects with advanced previously untreated non-small-cell lung tumors. J Clin Oncol 2000;18:1351-1359.

29 Rischin D, Peters L, Hicks R, et al. Phase I trial of concurrent tirapazamine, cisplatin, and radiotherapy inpatients with advanced head and neck cancer. J Clin Oncol 2001;19:535-542.

30 Treuner K, Findeisen M, Strausfeld U, et al. Phosphorylation of replication protein A middle subunit (RPA32) leads to a disassembly of the RPA heterotrimer. J Biol Chem 1999;274:15556-15561.

31 Jullien D, Gorlich D, Laemmli UK, et al. Nuclear import of RPA in Xenopus egg extracts requires a novel protein XRIPalpha but not importin alpha. EMBO J 1999;18:4348-4358.

32 Wang Y, Zhou XY, Wang H, et al. Roles of replication protein $A$ and DNA-dependent protein kinase in the regulation of DNA replication following DNA damage. J Biol Chem 1999;274:22060-22064.

33 Liu J-S, Kuo S-R, McHugh MM, et al. Adozelesin triggers DNA damage response pathways and arrests SV40 DNA replication through replication protein A inactivation. J Biol Chem 2000;275:1391-1397.

34 Riva F, Zuco V, Vink AA, et al. UV-induced DNA incision and proliferating cell nuclear antigen recruitment to repair sites occur independently of p53replication protein A interaction in p53 wild type and mutant ovarian carcinoma cells. Carcinogenesis 2002;22:1971-1978. 